\title{
Redefined soft relations and soft functions
}

\author{
Keyun Qin ${ }^{1 *}$ Qiong Liu ${ }^{1,2}$, Yang Xu${ }^{1}$ \\ ${ }^{1}$ College of Mathematics, Southwest Jiaotong University, Chengdu, Sichuan, 610031, China, \\ E-mail: keyunqin@263.net(Qin), \\ E-mail: lqhz1984@163.com(Liu),xuyang@home.swjtu.edu.cn(Xu) \\ ${ }^{2}$ School of Mathematics \& Computer Engineering, Xihua University, Chengdu, Sichuan, 610039, China
}

Received 31 July 2014

Accepted 2 June 2015

\begin{abstract}
Soft set theory, proposed by Molodtsov, has been regarded as an effective mathematical tool to deal with uncertainties. In this paper, the notion of soft relation is introduced which is a generalization of the notion of soft set relation, and some related properties are examined. Furthermore, the connections between soft relations and fuzzy sets are analyzed. It is shown that fuzzy relation and fuzzy soft set may be considered as special cases of soft relation.
\end{abstract}

Keywords: Soft sets, Fuzzy sets, Soft relations, Soft functions, Composition of soft relations

\section{Introduction}

To solve complicated problems in economics, engineering, environmental science and social science, methods in classical mathematics are not always successful because of various types of uncertainties present in these problems. While probability theory, fuzzy set theory ${ }^{1}$, rough set theory ${ }^{2,3}$, and other mathematical tools are well-known and often useful approaches to describe uncertainty, each of these theories has its inherent difficulties as pointed out $i^{4,5}$. Consequently, Molodtsov ${ }^{4}$ proposed a completely new approach for modeling vagueness and uncertainty in 1999. This approach called soft set theory is free from the difficulties affecting existing methods.

There is a growing interest in soft set theory. Maji et al. ${ }^{6}$ defined several operations on soft sets and made a theoretical study on the theory of soft sets. Based on ${ }^{6}$, Ali et al. ${ }^{7}$ introduced some new operations on soft sets and improved the notion of complement of soft set. They proved that certain De Morgans laws hold in soft set theory with respect to these new operations. The basic properties of operations on soft sets are analyzed systematically by Sezgin et al. ${ }^{8}$. Qin et al. ${ }^{9}$ introduced the notion of soft equality and established lattice structures and soft quotient algebras of soft sets. Xiao et al. ${ }^{10}$ proposed the notion of the exclusive disjunctive soft set and applied it to attribute reduction of incomplete information system. Gong et al. ${ }^{11}$ proposed the concept of the bijective soft set and some of its operations. Furthermore, soft set theory has been applied to several algebraic structures such as groups ${ }^{12}$, semirings ${ }^{13}$, rings ${ }^{14}, \mathrm{BCK} / \mathrm{BCI}-$ algebras ${ }^{15,16}$, and BL-algebras ${ }^{17}$. Also, the soft sets have been extended to fuzzy soft sets ${ }^{18,19}$, intuitionistic fuzzy soft sets ${ }^{20,21}$, interval-valued fuzzy soft sets ${ }^{22}$, vague soft sets ${ }^{23}$, interval-valued intuitionistic fuzzy soft $\operatorname{sets}^{24}$. The combination of

\footnotetext{
${ }^{*}$ Corresponding author.
} 
soft set and rough set has also been extensively investigated $^{25,26,27,28,29}$.

Recently, Babitha and Sunil ${ }^{30}$ proposed the notion of soft set relation and many related concepts such as equivalent soft set relation, partition, composition of soft set relations and soft set functions are discussed. It extends the notions of relations and functions to the framework of soft sets. Also the same authors ${ }^{31}$ introduced the notions of antisymmetric relation and transitive closure of a soft set relation. An algorithm is presented for calculating the transitive closure of a soft set relation. Based $\mathrm{on}^{30}$, Yang and Guo ${ }^{32}$ proposed the notions of antireflexive kernel, symmetric kernel, reflexive closure, and symmetric closure of a soft set relation. In the present paper, we attempt to conduct a further study along this line.

Babitha and Sunil ${ }^{30}$ initiated the theoretical aspects of soft sets by extending the notions of relations, equivalence relations, composition of relations and functions to the framework of soft sets. This study presents a preliminary, but potentially interesting research direction. This paper is devoted to a further discussion of soft set relation. The paper is organized as follows: In Section 2, we recall some notions and properties of soft sets and soft set relations. In Section 3, we propose the notion of soft relation which is a generalization of soft set relation presented $\mathrm{in}^{30}$. Some related concepts such as inverse soft relation, composition of soft relations and soft functions are introduced with their basic properties being discussed. In Section 4, we point out some interesting connections between soft relations and fuzzy sets. The paper is completed with some concluding remarks.

\section{Overview of soft sets and soft set relations}

In this section, we first recall some fundamental notions of soft sets and soft set relations ${ }^{4,6,7,30}$. Then we point out some limitations of the notion of soft set relation presented $\mathrm{in}^{30}$.

Let $U$ be the universe set and $E$ the set of all possible parameters under consideration with respect to $U$. Usually, parameters are attributes, characteristics, or properties of objects in $U$. Molodtsov ${ }^{4}$ de- fined the notion of a soft set in the following way:

Definition $1{ }^{4}$ A pair $(F, A)$ is called a soft set over $U$, where $A \subseteq E$ and $F$ is a mapping given by $F$ : $A \rightarrow P(U)$.

In other words, a soft set over $U$ is a parameterized family of subsets of $U$. For $e \in A, F(e)$ may be considered as the set of $e$-approximate elements of the soft set $(F, A)$. For illustration, Molodtsov considered several concrete examples of soft sets.

Example $1{ }^{4}$ Suppose that there are six houses in the universe $U$ given by $U=\left\{h_{1}, h_{2}, h_{3}, h_{4}, h_{5}, h_{6}\right\}$ and $E=\left\{e_{1}, e_{2}, e_{3}, e_{4}, e_{5}\right\}$ is the set of parameters. Where $e_{1}$ stands for the parameter 'expensive', $e_{2}$ stands for the parameter 'beautiful', $e_{3}$ stands for the parameter 'wooden', $e_{4}$ stands for the parameter 'cheap' and $e_{5}$ stands for the parameter 'in the green surroundings'.

In this case, to define a soft set means to point out expensive houses, beautiful houses, and so on. The soft set $(F, E)$ may describe the 'attractiveness of the houses' which Mr.X is going to buy. Suppose that $F\left(e_{1}\right)=\left\{h_{2}, h_{4}\right\}, F\left(e_{2}\right)=\left\{h_{1}, h_{3}\right\}, F\left(e_{3}\right)=\left\{h_{3}, h_{4}, h_{5}\right\}$, $F\left(e_{4}\right)=\left\{h_{1}, h_{3}, h_{5}\right\}, F\left(e_{5}\right)=\left\{h_{1}\right\}$. Then the soft set $(F, E)$ is a parameterized family $\left\{F\left(e_{i}\right) ; 1 \leqslant i \leqslant 5\right\}$ of subsets of $U$ and give us a collection of approximate descriptions of an object. $F\left(e_{1}\right)=\left\{h_{2}, h_{4}\right\}$ means 'houses $h_{2}$ and $h_{4}$ ' are 'expensive'.

Maji et al. ${ }^{6}$ made a theoretical study on the theory of soft sets. They introduced and investigated several operations on soft sets.

Definition $2{ }^{6}$ For two soft sets $(F, A)$ and $(G, B)$ over a common universe $U$, we say that $(F, A)$ is a soft subset of $(G, B)$ if

(1) $A \subset B$, and

(2) $\forall \epsilon \in A, F(\epsilon)$ and $G(\epsilon)$ are identical approximations.

We write $(F, A) \widetilde{\subset}(G, B)$.

Definition $3{ }^{6}$ For two soft sets $(F, A)$ and $(G, B)$ over a common universe $U$, the union of $(F, A)$ and $(G, B)$, denoted by $(F, A) \widetilde{\cup}(G, B)$, is the soft set $(H, C)$, where $C=A \cup B$, and $\forall e \in C$,

$$
H(e)= \begin{cases}F(e), & \text { if } e \in A-B, \\ G(e), & \text { if } e \in B-A, \\ F(e) \cup G(e), & \text { if } e \in A \cap B .\end{cases}
$$


Babitha and Sunil ${ }^{30}$ defined the notions of soft set relation and soft set function based on the Cartesian product of soft sets. It extends the notions of equivalence relations, composition of relations and functions to the framework of soft sets.

Definition $4{ }^{30}$ Let $(F, A)$ and $(G, B)$ be two soft sets over $U$, the Cartesian product of $(F, A)$ and $(G, B)$ is defined as $(F, A) \times(G, B)=(H, A \times B)$, where $H$ : $A \times B \rightarrow P(U \times U)$ is given by $H(a, b)=F(a) \times G(b)$ for all $(a, b) \in A \times B$.

Definition $5{ }^{30}$ Let $(F, A)$ and $(G, B)$ be two soft sets over $U$.

(1) A soft set relation from $(F, A)$ to $(G, B)$ is a soft subset of $(F, A) \times(G, B)$.

(2) A soft set relation from $(F, A)$ to $(F, A)$ is referred to as a soft set relation on $(F, A)$.

By this definition, a soft set relation from $(F, A)$ to $(G, B)$ is a soft set $(H, C)$, where $C \subseteq A \times B$ and $H(a, b)=F(a) \times G(b)$ for every $(a, b) \in C$. For illustration, Babitha and Sunil considered the following example.

Example $2{ }^{30}$ Let $U=\left\{p_{1}, p_{2}, p_{3}, p_{4}, p_{5}, p_{6}, p_{7}, p_{8}, p_{9}, p_{10}\right\}$ denote set of people in a social gathering. Let $A$ denote different job categories and take $A=\{c, d, e, t\}$, where $c, d, e$ and $t$ stand for chartered accountant, doctors, engineers and teachers respectively. Let $B$ denote the qualification of people and take $B=\left\{b_{1}, b_{2}, m_{1}, m_{2}\right\}$, where $b_{1}, b_{2}, m_{1}$ and $m_{2}$ stand for B.Sc., B.Tech., MBBS and M.Sc. respectively.

The soft set $(F, A)$ is given by $\left\{F(c)=\left\{p_{1}, p_{2}\right\}\right.$, $\left.F(d)=\left\{p_{4}, p_{5}\right\}, F(e)=\left\{p_{7}, p_{9}\right\}, F(t)=\left\{p_{3}, p_{4}, p_{7}\right\}\right\}$ and it describes people having different jobs. The soft set $(G, B)$ is given by $\left\{G\left(b_{1}\right)=\left\{p_{1}, p_{6}, p_{8}, p_{10}\right\}\right.$, $G\left(b_{2}\right)=\left\{p_{3}, p_{6}, p_{7}, p_{9}\right\}, \quad G\left(m_{1}\right)=\left\{p_{3}, p_{4}, p_{5}, p_{8}\right\}$, $\left.G\left(m_{2}\right)=\left\{p_{3}, p_{8}\right\}\right\}$ and it represents the people qualified in various courses.

Define a soft set relation $R=(H, C)$ from $(F, A)$ to $(G, B)$ by $C=\left\{\left(d, m_{1}\right),\left(e, b_{2}\right)\right\}, H\left(d, m_{1}\right)=F(d) \times$ $G\left(m_{1}\right)$, and $H\left(e, b_{2}\right)=F(e) \times G\left(b_{2}\right)$.

In this example, by $F(d) \subseteq G\left(m_{1}\right)$ and $F(e) \subseteq$ $G\left(b_{2}\right), H\left(d, m_{1}\right)=F(d) \times G\left(m_{1}\right)$ means the people who is s doctor must have a MBBS degree, and $H\left(e, b_{2}\right)=F(e) \times G\left(b_{2}\right)$ means every engineer must have a B.Tech. degree.

Theoretically speaking, a soft set relation from $(F, A)$ to $(G, B)$ may be looked upon as a subset of
$\{F(a) \times G(b) ; a \in A, b \in B\}$ We consider a simple situation where $A=\{a\}, B=\{b\}$ are sets with single element. In this case, a soft set relation from $(F, A)$ to $(G, B)$ is $F(a) \times G(b)$ if it is nonempty. From Cantor's set theory we know that any subsets of $F(a) \times G(b)$ is a binary relation from $F(a)$ to $G(b)$. It follows that in this case the notion of soft set relation represents an extreme situation (i.e., the whole relation $F(a) \times G(b)$ from $F(a)$ to $G(b))$. Thus it seems that revising the condition ' $H(a, b)=F(a) \times G(b)^{\prime}$ in the definition of soft set relation to ' $H(a, b) \subseteq$ $F(a) \times G(b)^{\prime}$ may lead to a new definition of soft set relation which is consistent with set theory.

Ali et al. ${ }^{7}$ note that if $(F, A)$ and $(G, B)$ are two different soft sets, then it is not necessary for these two soft sets have the same approximation subset of $U$ for a particular common parameter say $c \in A \cap B$, i.e., $F(c) \neq G(c)$ in general. Based on this observation, they introduced some new operations on soft sets.

Definition $6{ }^{7}$ Let $(F, A)$ and $(G, B)$ be two soft sets over a common universe $U$.

(1) The extended intersection of $(F, A)$ and $(G, B)$, denoted by $(F, A) \sqcap_{\varepsilon}(G, B)$, is the soft set $(H, C)$, where $C=A \cup B$, and $\forall e \in C$,

$$
H(e)= \begin{cases}F(e), & \text { if } e \in A-B, \\ G(e), & \text { if } e \in B-A, \\ F(e) \cap G(e), & \text { if } e \in A \cap B .\end{cases}
$$

(2) The restricted intersection of $(F, A)$ and $(G, B)$, denoted by $(F, A) \cap(G, B)$, is the soft set $(H, C)$, where $C=A \cap B$, and $\forall e \in C, H(e)=F(e) \cap$ $G(e)$.

(3) The restricted union of $(F, A)$ and $(G, B)$, denoted by $(F, A) \cup_{\mathfrak{R}}(G, B)$, is the soft set $(H, C)$, where $C=A \cap B$, and $\forall e \in C, H(e)=F(e) \cup G(e)$.

Based on these operations, Qin et al..$^{9}$ established the lattice structures of soft sets.

Theorem $1{ }^{9}$ (1) $(S(U, E), \widetilde{\cup}$, ก) is a bounded distributive lattice, where

$S(U, E)=\{(F, A) ; A \subseteq E, F: A \rightarrow P(U)\}$

is the set of all soft sets over the universe $U$ and the parameter set $E$.

(2) Let $\leqslant$ be the ordering relation in lattice $(S(U, E), \widetilde{\cup}$, ก $)$ and $(F, A),(G, B) \in S(U, E) .(F, A) \leqslant$ 
$(G, B)$ if and only if $A \subseteq B$ and $F(e) \subseteq G(e)$ for all $e \in A$.

This theorem lead to a new definition of soft subset which is different from Definition 2.

Definition $7{ }^{26,33}$ For two soft sets $(F, A)$ and $(G, B)$ over a common universe $U$, we say that $(F, A)$ is a soft subset of $(G, B)$, denoted by $(F, A) \subseteq(G, B)$, if $A \subseteq B$ and $F(e) \subseteq G(e)$ for every $e \in A$.

Remark: In this definition, we use the symbol $\subseteq$ to denote soft subset relation to differentiate it from Definition 2. In the next section, we adopt this new definition of soft subset to discuss soft relations and soft functions.

\section{Soft relations and soft functions}

In this section, we extend the notions of soft set relation and soft set function presented in $^{30}$ and investigate some related concepts. We first extend the notion of Cartesian product to soft sets over different universes.

Definition 8 Let $(F, A)$ be a soft set over the universe $U$ and $(G, B)$ a soft set over the universe $V$. The Cartesian product of $(F, A)$ and $(G, B)$ is a soft set over $U \times V$ and is defined as $(F, A) \times(G, B)=$ $(H, A \times B)$, where $H: A \times B \rightarrow P(U \times V)$ is given by $H(a, b)=F(a) \times G(b)$ for all $(a, b) \in A \times B$.

Definition 9 Let $(F, A)$ be a soft set over the universe $U$ and $(G, B)$ a soft set over the universe $V$.

(1) If $(H, C) \subseteq(F, A) \times(G, B)$, i.e., $C \subseteq A \times B$ and $H(a, b) \subseteq F(a) \times G(b)$ for each $(a, b) \in C$, then $(H, C)$ is called a soft relation from $(F, A)$ to $(G, B)$.

(2) A soft relation from $(F, A)$ to $(F, A)$ is called a soft relation on $(F, A)$.

From this definition, $(H, C)$ is a soft relation from $(F, A)$ to $(G, B)$ if and only if $C$ is a classical binary relation from $A$ to $B$ and $H(a, b)$ is a classical binary relation from $F(a)$ to $G(b)$ for every $(a, b) \in C$. Clearly, the notion of soft relation is a generalization of soft set relation given by Definition 5 .

Example 3 Let $(F, A)$ be the soft set given in Example 2. It represents people having different jobs.

Suppose that $V=\left\{h_{1}, h_{2}, h_{3}, h_{4}, h_{5}, h_{6}, h_{7}\right\}$ is a set of houses and $B=\left\{e_{1}, e_{2}, e_{3}\right\}$ is the set of parameters, where $e_{1}, e_{2}$ and $e_{3}$ stand for 'wooden', 'beau- tiful' and 'in the green surroundings' respectively. Assume the soft set $(G, B)$ is given by $\left\{G\left(e_{1}\right)=\right.$ $\left.\left\{h_{1}, h_{2}, h_{6}\right\}, G\left(e_{2}\right)=\left\{h_{1}, h_{3}, p_{4}\right\}, G\left(e_{3}\right)=\left\{h_{5}, h_{7}\right\}\right\}$. It describes the attractiveness of the houses.

We suppose that $p_{1}, p_{2}$ and $p_{9}$ are going to buy a house. Different person may be interested in different characteristics of the houses. In addition, for a particular house, each person has his/her own opinion and the evaluations given by different persons may be extremely different. Assume that:

$p_{1}$ is interested in buying the wooden house $h_{1}$, or $h_{2}$, or the house $h_{5}$ which is in green surroundings;

$p_{2}$ is interested in buying the wooden house $h_{2}$, or $h_{6}$;

$p_{9}$ is interested in buying the beautiful house $h_{3}$.

Let $(p, h)$ denote the fact that person $p$ is interested in buying the house $h$. It forms a soft relation $(H, C)$ from $(F, A)$ to $(G, B)$, where $C=$ $\left\{\left(c, e_{1}\right),\left(c, e_{3}\right),\left(e, e_{2}\right)\right\} \subseteq A \times B$, and

$H\left(c, e_{1}\right)=\left\{\left(p_{1}, h_{1}\right),\left(p_{1}, h_{2}\right),\left(p_{2}, h_{2}\right),\left(p_{2}, h_{6}\right)\right\} \subseteq$ $F(c) \times G\left(e_{1}\right)$;

$H\left(c, e_{3}\right)=\left\{\left(p_{1}, h_{5}\right)\right\} \subseteq F(c) \times G\left(e_{3}\right) ;$

$H\left(e, e_{2}\right)=\left\{\left(p_{9}, h_{3}\right)\right\} \subseteq F(e) \times G\left(e_{2}\right)$.

This soft relation represents to some extent some persons' desire for buying houses.

\subsection{Compositions of soft relations}

Definition 10 Let $(F, A),(G, B)$ and $(H, C)$ be three soft sets. Let $\Re=(R, D)$ be a soft relation from $(F, A)$ to $(G, B)$ and $\subseteq=(S, K)$ be a soft relation from $(G, B)$ to $(H, C)$. The composition of $\Re$ and $\subseteq$, denoted by $\subseteq \circ \Re$, is a soft relation $\subseteq \circ \Re=(L, K \circ D)$ from $(F, A)$ to $(H, C)$ given by:

$K \circ D=\{(a, c) \in A \times C ; \exists b \in B((a, b) \in D \wedge(b, c) \in$ $K)\}$,

$L(a, c)=\cup_{b \in E(a, c)} S(b, c) \circ R(a, b)=\cup_{b \in E(a, c)}\{(x, z) ;$

$\exists y((x, y) \in R(a, b) \wedge(y, z) \in S(b, c))\}$

for each $(a, c) \in K \circ D$, where $E(a, c)=\{b \in B ;(a, b) \in$ $D,(b, c) \in K\}$.

In this definition, $K \circ D$ is the composition of classical relations $D$ and $K$. For each $(a, c) \in K \circ D$ and $b \in E(a, c)$, by $(a, b) \in D$ and $(b, c) \in K$ we know that $R(a, b) \subseteq F(a) \times G(b)$ is a classical relation from $F(a)$ to $G(b)$ and $S(b, c) \subseteq G(b) \times H(c)$ 
is a classical relation from $G(b)$ to $H(c) . S(b, c) \circ$ $R(a, b)=\{(x, z) ; \exists y((x, y) \in R(a, b) \wedge(y, z) \in S(b, c))\}$ is the composition of $R(a, b)$ and $S(b, c)$. It follows that $S(b, c) \circ R(a, b) \subseteq F(a) \times H(c)$ is a classical relation from $F(a)$ to $H(c)$. This implies that $\subseteq \circ \Re$ is indeed a soft relation from $(F, A)$ to $(H, C)$.

Definition 11 Let $(F, A)$ and $(G, B)$ be soft sets and $\Re=(R, D)$ a soft relation from $(F, A)$ to $(G, B)$. The inverse soft relation of $\Re$ is a soft relation from $(G, B)$ to $(F, A)$ given by $\Re^{-1}=\left(R^{-1}, D^{-1}\right)$, where $D^{-1}=\{(b, a) ;(a, b) \in D\}$ and $R^{-1}(b, a)=(R(a, b))^{-1}$ are the inverse relations of $D$ and $R(a, b)$, respectively.

Theorem 2 Let $(F, A),(G, B),(H, C)$ be soft sets, $\Re=(R, D)$ a soft relation from $(F, A)$ to $(G, B)$ and $\subseteq=(S, K)$ a soft relation from $(G, B)$ to $(H, C)$. Then $(\mathfrak{S} \circ \mathfrak{R})^{-1}=\mathfrak{R}^{-1} \circ \mathfrak{S}^{-1}$.

Proof. Let $\subseteq \circ \Re=(L, K \circ D)$ be the composition of $\Re$ and $\mathfrak{S}$. It follows that $(\mathfrak{S} \circ \Re)^{-1}=\left(L^{-1},(K \circ\right.$ $\left.D)^{-1}\right)=\left(L^{-1}, D^{-1} \circ K^{-1}\right)$ is a soft relation. For each $(c, a) \in D^{-1} \circ K^{-1}$, we have

$L^{-1}(c, a)=(L(a, c))^{-1}$

$=\left(\cup_{b \in E(a, c)} S(b, c) \circ R(a, b)\right)^{-1}$

$=\cup_{b \in E(a, c)}(S(b, c) \circ R(a, b))^{-1}$

$=\cup_{b \in E(a, c)}(R(a, b))^{-1} \circ(S(b, c))^{-1}$

$=\cup_{b \in E(a, c)} R^{-1}(b, a) \circ S^{-1}(c, b)$.

On the other hand, $\Re^{-1} \circ \mathfrak{S}^{-1}=\left(M, D^{-1} \circ\right.$ $\left.K^{-1}\right)$ and for each $(c, a) \in D^{-1} \circ K^{-1}, M(c, a)=$ $\cup_{b \in E(c, a)} R^{-1}(b, a) \circ S^{-1}(c, b)$.

Furthermore, for each $(c, a) \in D^{-1} \circ K^{-1}$ and $b \in$ $B$, we have

$b \in E(a, c) \Leftrightarrow(a, b) \in D \wedge(b, c) \in K \Leftrightarrow(c, b) \in$ $K^{-1} \wedge(b, a) \in D^{-1} \Leftrightarrow b \in E(c, a)$.

It follows that $E(a, c)=E(c, a)$ and consequently, $L^{-1}(c, a)=M(c, a)$. Thus $(\mathfrak{S} \circ \Re)^{-1}=\mathfrak{R}^{-1} \circ \mathfrak{S}^{-1}$.

Definition 12 Let $\Re=(R, D)$ be a soft relation on $(F, A)$.

(1) $\Re$ is called an reflexive soft relation if $D$ is a classical reflexive relation on $A$ and $R(a, a)$ is a classical reflexive relation on $F(a)$ for each $a \in A$.

(2) $\Re$ is called a symmetric soft relation if $D$ is a classical symmetric relation on $A$ and $R(a, b)=$ $(R(b, a))^{-1}$ for each $(a, b) \in D$.

(3) $R$ is called a transitive soft relation if $D$ is a classical transitive relation on $A$ and $R(b, c) \circ$
$R(a, b) \subseteq R(a, c)$ for each $(a, b),(b, c) \in D$.

Theorem 3 Let $\Re=(R, D)$ be a soft relation on $(F, A)$.

(1) $\Re$ is symmetric if and only if $\Re=\Re^{-1}$.

(2) $\Re$ is transitive if and only if $\Re \circ \Re \subseteq \Re$.

Proof. (1) $\Re=\Re^{-1} \Leftrightarrow D=D^{-1} \wedge R=R^{-1} \Leftrightarrow D$ is symmetric and $R(a, b)=R^{-1}(a, b)$ for each $(a, b) \in$ $D \Leftrightarrow D$ is symmetric and $R(a, b)=(R(b, a))^{-1}$ for each $(a, b) \in D \Leftrightarrow \Re$ is symmetric.

(2) Let $\Re \circ \Re=(L, D \circ D)$. Assume that $\Re$ is transitive. It follows that $D$ is transitive and hence $D \circ D \subseteq D$. For each $(a, c) \in D \circ D$ and $b \in E(a, c)$, we have $(a, b) \in D,(b, c) \in D$ and hence $R(b, c) \circ R(a, b) \subseteq$ $R(a, c)$ by the definition. Consequently,

$L(a, c)=\cup_{b \in E(a, c)} R(b, c) \circ R(a, b) \subseteq R(a, c)$.

It follows that $\Re \circ \Re$ is a soft subset of $\Re$, i.e., $\Re \circ \Re \subseteq \Re$.

Conversely, assume that $\mathfrak{R} \circ \mathfrak{R} \subseteq \Re$. It follows that $D \circ D \subseteq D$ and $L(a, c) \subseteq R(a, c)$ for each $(a, c) \in$ $D \circ D$. By $D \circ D \subseteq D$ we know that $D$ is transitive. For each $(a, b),(b, c) \in D$, we have $b \in E(a, c)$ and hence

$$
\begin{aligned}
& R(b, c) \circ R(a, b) \subseteq \cup_{e \in E(a, c)} R(e, c) \circ R(a, e) \\
& =L(a, c) \subseteq R(a, c) .
\end{aligned}
$$

Thus $\Re$ is transitive.

\subsection{Soft functions}

Definition 13 Let $(F, A)$ and $(G, B)$ be soft sets over the universe $U$ and $V$ respectively, $f=(H, C)$ a soft relation from $(F, A)$ to $(G, B)$. $f$ is called a soft function if

(1) $\left(a, b_{1}\right) \in C$ and $\left(a, b_{2}\right) \in C$ imply $b_{1}=b_{2}$ for each $a \in A$ and $b_{1}, b_{2} \in B$;

(2) $\left(x, y_{1}\right) \in H(a, b)$ and $\left(x, y_{2}\right) \in H(a, b)$ imply $y_{1}=y_{2}$ for each $(a, b) \in C, x \in U$ and $y_{1}, y_{2} \in V$.

In this definition, $C$ is a partial function from $A$ to $B$. Let $A_{1}=\{a \in A ; \exists b \in B((a, b) \in C)\}$ and $B_{1}=\{b \in B ; \exists a \in A((a, b) \in C)\}$. It follows that $C$ is a surjective function from $A_{1}$ to $B_{1} . A_{1}$ and $B_{1}$ are domain and range of $C$ respectively. If $(a, b) \in C$, then we write $C(a)=b$. Similarly, for each $a \in$ $A_{1}, H(a, C(a))$ is a partial function from $F(a)$ to $G(C(a))$. The domain of $H(a, C(a))$ is $\{x \in F(a) ; \exists y \in$ $V((x, y) \in H(a, C(a)))\}$ and the range of $H(a, C(a))$ is 
$\{y \in G(C(a)) ; \exists x \in U((x, y) \in H(a, C(a)))\}$. If $(x, y) \in$ $H(a, C(a))$, then we write $H(a, C(a))(x)=y$.

Example 4 Let $U=\left\{p_{1}, p_{2}, p_{3}, p_{4}, p_{5}, p_{6}\right\}, \quad A=$ $\left\{a_{1}, a_{2}, a_{3}, a_{4}\right\}, B=\left\{b_{1}, b_{2}\right\}$. Consider the soft sets $(F, A)$ and $(G, B)$ defined by $F\left(a_{1}\right)=\left\{p_{1}, p_{2}, p_{5}\right\}$, $F\left(a_{2}\right)=\left\{p_{2}, p_{3}, p_{4}\right\}, F\left(a_{3}\right)=\left\{p_{1}, p_{2}\right\}$ and $G\left(b_{1}\right)=$ $\left\{p_{1}, p_{2}\right\}, G\left(b_{2}\right)=\left\{p_{2}, p_{5}, p_{6}\right\}$.

(1) Let $f=(H, C), C=\left\{\left(a_{1}, b_{1}\right),\left(a_{2}, b_{1}\right),\left(a_{3}, b_{2}\right),\left(a_{4}, b_{2}\right)\right\}$, $H\left(a_{1}, b_{1}\right)=F\left(a_{1}\right) \times G\left(b_{1}\right), H\left(a_{2}, b_{1}\right)=F\left(a_{2}\right) \times G\left(b_{1}\right)$, $H\left(a_{3}, b_{2}\right)=F\left(a_{3}\right) \times G\left(b_{2}\right), H\left(a_{4}, b_{2}\right)=F\left(a_{4}\right) \times G\left(b_{2}\right)$. It follows that $f$ is a soft set function in the sense of Definition 5.1 $1^{30}$. By $H\left(a_{1}, b_{1}\right)=F\left(a_{1}\right) \times G\left(b_{1}\right)=$ $\left\{p_{1}, p_{2}, p_{5}\right\} \times\left\{p_{1}, p_{2}\right\}$, we have $\left(p_{5}, p_{1}\right),\left(p_{5}, p_{2}\right) \in$ $H\left(a_{1}, b_{1}\right)$. Hence $f$ is not a soft function.

(2) Let $g=(R, C)$, and

$R\left(a_{1}, b_{1}\right)=\left\{\left(p_{1}, p_{1}\right),\left(p_{2}, p_{2}\right)\right\} \subseteq F\left(a_{1}\right) \times G\left(b_{1}\right)$,

$R\left(a_{2}, b_{1}\right)=\left\{\left(p_{2}, p_{1}\right),\left(p_{4}, p_{2}\right)\right\} \subseteq F\left(a_{2}\right) \times G\left(b_{1}\right)$,

$R\left(a_{3}, b_{2}\right)=\left\{\left(p_{1}, p_{2}\right),\left(p_{2}, p_{2}\right)\right\} \subseteq F\left(a_{3}\right) \times G\left(b_{2}\right)$,

$R\left(a_{4}, b_{2}\right)=\left\{\left(p_{5}, p_{5}\right),\left(p_{6}, p_{2}\right)\right\} \subseteq F\left(a_{4}\right) \times G\left(b_{2}\right)$.

Then $g$ is a soft function from $(F, A)$ to $(G, B)$.

Definition 14 Let $(F, A)$ and $(G, B)$ be soft sets over the universe $U$ and $V$ respectively, $f=(H, C)$ a soft function from $(F, A)$ to $(G, B)$.

(1) The domain of $f$, denoted by $\operatorname{dom}(f)$, is defined as the soft set $\left(D, A_{1}\right)$, where $D(a)$ is the projection of $H(a, C(a))$ on $U$, i.e.,

$D(a)=\{x \in U ; \exists y \in V((x, y) \in H(a, C(a)))\}$,

for each $a \in A_{1}$.

(2) The range of $f$, denoted by $\operatorname{ran}(f)$, is defined as the soft set $\left(R, B_{1}\right)$, where

$R(b)=\left\{y \in V ; \exists a \in A_{1} \exists x \in U(C(a)=b \wedge\right.$ $H(a, C(a))(x)=y)\}$,

for each $b \in B_{1}$.

Clearly, $\operatorname{dom}(f)$ and $\operatorname{ran}(f)$ are soft subsets of $(F, A)$ and $(G, B)$ respectively. Furthermore,

$R(b)=\cup_{a \in C^{-1}(b)}\{y \in V ; \exists x \in U(H(a, b)(x)=y)\}$, where $C^{-1}(b)=\left\{a \in A_{1} ; C(a)=b\right\}$.

Theorem 4 Let $(F, A),(G, B),(H, C)$ be soft sets, $f=(R, D)$ a soft function from $(F, A)$ to $(G, B)$ and $g=(S, K)$ a soft function from $(G, B)$ to $(H, C)$. Then $g \circ f$ is a soft function from $(F, A)$ to $(H, C)$.

Proof. Let $g \circ f=(L, K \circ D)$. Assume that $\left(a, c_{1}\right),\left(a, c_{2}\right) \in K \circ D$. It follows that there exist $b_{1}, b_{2} \in B$ such that $\left(a, b_{1}\right) \in D,\left(b_{1}, c_{1}\right) \in K,\left(a, b_{2}\right) \in$ $D$ and $\left(b_{2}, c_{2}\right) \in K$. By $\left(a, b_{1}\right) \in D$ and $\left(a, b_{2}\right) \in D$, $b_{1}=b_{2}$ followed. Consequently, $c_{1}=c_{2}$ follows from $\left(b_{1}, c_{1}\right) \in K$ and $\left(b_{1}, c_{2}\right) \in K$.

Assume that $(a, c) \in K \circ D$. Since $D$ is a function, there exists unique $b \in B$ such that $(a, b) \in D$, $(b, c) \in K$. It follows that $L(a, c)=S(b, c) \circ R(a, b)$. Suppose that $\left(x, z_{1}\right),\left(x, z_{2}\right) \in L(a, c)$. Then there exist $y_{1}, y_{2}$ such that $\left(x, y_{1}\right) \in R(a, b),\left(y_{1}, z_{1}\right) \in S(b, c)$, $\left(x, y_{2}\right) \in R(a, b)$ and $\left(y_{2}, z_{2}\right) \in S(b, c)$. It follows that $y_{1}=y_{2}$ and consequently $z_{1}=z_{2}$.

Definition 15 Let $(F, A)$ and $(G, B)$ be soft sets over the universe $U$ and $V$ respectively. A soft function $f=(H, C)$ from $(F, A)$ to $(G, B)$ is called injective (one-one) if

(1) $C\left(a_{1}\right)=C\left(a_{2}\right)$ implies $a_{1}=a_{2}$ for every $a_{1}, a_{2} \in A$;

(2) $H(a, b)\left(x_{1}\right)=H(a, b)\left(x_{2}\right)$ implies $x_{1}=x_{2}$ for every $(a, b) \in C$ and $x_{1}, x_{2} \in U$.

Theorem 5 Let $(F, A)$ and $(G, B)$ be soft sets over the universe $U$ and $V$ respectively, $f=(H, C)$ an injective soft function from $(F, A)$ to $(G, B)$. Then $f^{-1}$ is a soft function from $(G, B)$ to $(F, A)$.

Proof. By Definition $18, f^{-1}=\left(H^{-1}, C^{-1}\right)$ is a soft relation from $(G, B)$ to $(F, A)$. Assume that $\left(b, a_{1}\right) \in C^{-1},\left(b, a_{2}\right) \in C^{-1}$. It follows that $\left(a_{1}, b\right) \in$ $C,\left(a_{2}, b\right) \in C$. Since $f$ is injective, we have $a_{1}=a_{2}$.

Assume that $(b, a) \in C^{-1}$ and $\left(y, x_{1}\right) \in$ $H^{-1}(b, a),\left(y, x_{2}\right) \in H^{-1}(b, a)$. It follows that $\left(y, x_{1}\right) \in$ $(H(a, b))^{-1},\left(y, x_{2}\right) \in(H(a, b))^{-1}$ and consequently $\left(x_{1}, y\right) \in H(a, b),\left(x_{2}, y\right) \in H(a, b)$. Since $f$ is injective, we have $x_{1}=x_{2}$ as required.

\section{Connections between soft relations and fuzzy sets}

The theory of fuzzy sets initiated by Zadeh ${ }^{1}$ provides an appropriate framework for representing and processing vague concepts by allowing partial memberships. Let $U$ be a nonempty set, called universe. A fuzzy set $\mu$ on $U$ is defined by a membership function $\mu: U \rightarrow[0,1]$. For $x \in U$, the membership value $\mu(x)$ essentially specifies the degree to which $x$ belongs to the fuzzy set $\mu$. We denote by $F(U)$ the set of all fuzzy sets on $U$.

There are many different definitions for fuzzy set 
operations. With the min-max system proposed by Zadeh $^{1}$, fuzzy set intersection and union are defined componentwise as follows:

$(\mu \cap \nu)(x)=\mu(x) \wedge v(x)$,

$(\mu \cup v)(x)=\mu(x) \vee v(x)$,

where $\mu, v$ are fuzzy sets on $U$ and $x \in U$.

Molodtsov $^{4}$ pointed out that fuzzy set may be considered as a special case of the soft set. Let $\mu$ be a fuzzy set on $U$. For $\alpha \in[0,1]$, let

$$
F(\alpha)=\{x \in U ; \mu(x) \geqslant \alpha\}
$$

be the $\alpha$-level set. If we know the family $\{F(\alpha) ; \alpha \in$ $[0,1]\}$, we can calculate $\mu(x)$ by means of the formulae $\mu(x)=\sup _{x \in F(\alpha)} \alpha$. That is $\mu=\cup_{\alpha \in[0,1]} \alpha$. $F(\alpha)$, where $\alpha \cdot F(\alpha)$ is a fuzzy set defined by $(\alpha$. $F(\alpha))(x)=\alpha$ if $x \in F(\alpha)$, and $(\alpha \cdot F(\alpha))(x)=0$ otherwise. This observation is usually summarized by a representation theorem in fuzzy set theory, which establishes a one-to-one correspondence between a fuzzy set and a family of crisp sets satisfying certain conditions. Thus, fuzzy set $\mu$ may be considered as the soft set $(F,[0,1])$.

Assume that $\mu, v$ are fuzzy sets on the universe $U$. Let $\left(F_{\mu},[0,1]\right)$ and $\left(F_{\nu},[0,1]\right)$ be soft sets induced by $\mu$ and $v$ respectively, i.e.,

$$
\begin{aligned}
& F_{\mu}(\alpha)=\{x \in U ; \mu(x) \geqslant \alpha\}, \\
& F_{v}(\alpha)=\{x \in U ; v(x) \geqslant \alpha\},
\end{aligned}
$$

for each $\alpha \in[0,1]$. We consider a soft relation $(H, C)$ from $\left(F_{\mu},[0,1]\right)$ to $\left(F_{v},[0,1]\right)$. By the definition, we have $C \subseteq[0,1] \times[0,1]$ and for all $\alpha, \beta \in[0,1]$,

$$
\begin{aligned}
& H(\alpha, \beta) \subseteq F_{\mu}(\alpha) \times F_{v}(\beta) \\
& =\{(x, y) \in U \times U ; \mu(x) \geqslant \alpha, v(y) \geqslant \beta\} . \\
& (1) \text { Let } C=\{(\alpha, \alpha) ; \alpha \in[0,1]\} \text { and } \\
& H(\alpha, \alpha)=F_{\mu}(\alpha) \times F_{v}(\alpha) \\
& =\{(x, y) \in U \times U ; \mu(x) \geqslant \alpha, v(y) \geqslant \alpha\}
\end{aligned}
$$

for each $\alpha \in[0,1]$. $(H, C)$ is a soft set over the universe $U \times U$. It is trivial that $\alpha \leqslant \beta$ implies $H(\beta, \beta) \subseteq H(\alpha, \alpha)$. Thus $(H, C)$ may be considered as a fuzzy set $\varepsilon$ on the universe $U \times U$ given by $\varepsilon=\cup_{\alpha \in[0,1]} \alpha \cdot H(\alpha, \alpha)$. For every $x, y \in U$, we have

$$
\begin{aligned}
& \varepsilon(x, y)=\vee_{\alpha \in[0,1]} \alpha \cdot H(\alpha, \alpha)(x, y) \\
& =\vee_{(x, y) \in H(\alpha, \alpha)} \alpha=\mu(x) \wedge v(y) .
\end{aligned}
$$

In fact, if $(x, y) \in H(\alpha, \alpha)$, then $\mu(x) \geqslant \alpha, v(y) \geqslant \alpha$ and hence $\mu(x) \wedge v(y) \geqslant \alpha$. Consequently, we have $\varepsilon(x, y)=\vee_{(x, y) \in H(\alpha, \alpha)} \alpha \leqslant \mu(x) \wedge \nu(y)$.

Conversely, let $\mu(x) \wedge v(y)=\alpha_{0}$. It follows that $\mu(x) \geqslant \alpha_{0}, v(y) \geqslant \alpha_{0}$ and hence $(x, y) \in H\left(\alpha_{0}, \alpha_{0}\right)$.
Thus we have $\mu(x) \wedge v(y)=\alpha_{0} \leqslant \vee_{(x, y) \in H(\alpha, \alpha)} \alpha=$ $\varepsilon(x, y)$.

Let $\gamma$ be the fuzzy set on $U$ given by $\gamma(x)=\varepsilon(x, x)$ for each $x \in U$. It follows that $\gamma=\mu \cap \nu$.

(2) Let $C=[0,1] \times[0,1]=\{(\alpha, \beta) ; \alpha, \beta \in[0,1]\}$ and

$$
\begin{aligned}
& H(\alpha, \beta)=\{x ; \mu(x)=\alpha\} \times\{y ; v(y)=\beta\} \\
& =\{(x, y) \in U \times U ; \mu(x)=\alpha, v(y)=\beta\}
\end{aligned}
$$

for each $\alpha, \beta \in[0,1] . \quad(H, C)$ is a soft set over the universe $U \times U$. Let $\varepsilon=\cup_{\alpha, \beta \in[0,1]}(\alpha \vee \beta) \cdot H(\alpha, \beta)$. It follows that $\varepsilon$ is a fuzzy set on $U \times U$. For every $x, y \in U$, we have

$$
\begin{aligned}
& \varepsilon(x, y)=\vee_{\alpha, \beta \in[0,1]}(\alpha \vee \beta) \cdot H(\alpha, \beta)(x, y) \\
& =\vee_{(x, y) \in H(\alpha, \beta)} \alpha \vee \beta=\mu(x) \vee v(y) .
\end{aligned}
$$

In fact, for every $x, y \in U$, let $\mu(x)=\alpha_{0}, v(y)=\beta_{0}$. It follows that $(x, y) \in H\left(\alpha_{0}, \beta_{0}\right)$ and $(x, y) \notin H(\alpha, \beta)$ if $\alpha \neq \alpha_{0}$ or $\beta \neq \beta_{0}$. Consequently, we have

$$
\varepsilon(x, y)=\vee_{(x, y) \in H(\alpha, \beta)} \alpha \vee \beta=\alpha_{0} \vee \beta_{0}=\mu(x) \vee v(y) .
$$

Let $\gamma$ be the fuzzy set on $U$ given by $\gamma(x)=\varepsilon(x, x)$ for each $x \in U$. It follows that $\gamma=\mu \cup v$.

(3) Let $R \in F(U \times V)$ be a fuzzy relation from $U$ to $V$. The projection of $R$ on $U$ and $V$ are denoted by $R_{\mu}$ and $R_{\nu}$ respectively. $R_{\mu}$ is a fuzzy set on $U$ given by

$R_{\mu}(x)=\vee_{y \in V} R(x, y), \forall x \in U$,

and $R_{v}$ is a fuzzy set on $V$ given by

$R_{v}(y)=\vee_{x \in U} R(x, y), \forall y \in V$.

It follows that $R_{\mu}=\left(F_{R_{\mu}},[0,1]\right), R_{v}=\left(F_{R_{v}},[0,1]\right)$ and $R=\left(F_{R},[0,1]\right)$ are soft sets over $U, V$ and $U \times V$ respectively, and for each $\alpha \in[0,1]$,

$F_{R_{\mu}}(\alpha)=\left\{x \in U ; R_{\mu}(x) \geqslant \alpha\right\}$,

$F_{R_{\nu}}(\alpha)=\left\{y \in V ; R_{v}(y) \geqslant \alpha\right\}$,

$F_{R}(\alpha)=\{(x, y) \in U \times V ; R(x, y) \geqslant \alpha\}$.

We assume that $(x, y) \in F_{R}(\alpha)$. It follows that $R(x, y) \geqslant \alpha$ and hence $R_{\mu}(x) \geqslant \alpha, R_{\gamma}(y) \geqslant \alpha$. Consequently, we have $F_{R}(\alpha) \subseteq F_{R_{\mu}}(\alpha) \times F_{R_{\nu}}(\alpha)$. That is to say, $R$ is a soft relation from $R_{\mu}$ to $R_{\nu}$.

(4) Let $S=(F, A)$ be a fuzzy soft set over $U$, i.e., $F(a)$ be a fuzzy set on $U$ for every $a \in A$. $S$ induces a fuzzy relation $R_{S}$ from $E$ to $U$ which is defined as: $R_{S}(a, x)=F(a)(x)$ if $a \in A$ and $R_{S}(a, x)=0$ otherwise, for every $a \in E$ and $x \in U$. Conversely, let $R$ be a fuzzy relation from $E$ to $U . R$ induces a fuzzy soft set $S_{R}=(F, A)$ over $U$ which is defined as: $A=\{a \in E ; \exists x \in U(R(a, x) \neq 0)\}, F(a)(x)=R(a, x)$ 
for every $a \in A, x \in U$. It follows that there exists a one-to-one correspondence between the set of all fuzzy soft sets over $U$ and the set of all fuzzy relations from $E$ to $U$. By (3), fuzzy soft set may be considered as a special case of soft relation.

From above observations, we know that the intersection and union operations on fuzzy sets can be characterized by soft relations. In addition, fuzzy relation and soft fuzzy set may be considered as special cases of soft relation. For illustration, we consider the following example.

Example 5 Let $U=\{x, y, z\}, V=\{a, b, c\}$ and $R \in$ $F(U \times V)$ be given by $R=0 /(x, a)+0.3 /(x, b)+$ $0.5 /(x, c)+0 /(y, a)+0.5 /(y, b)+0.5 /(y, c)+1 /(z, a)+$ $0.8 /(z, b)+0.2 /(z, c)$. It follows that $R_{\mu}=0.5 / x+$ $0.5 / y+1 / z$ and $R_{v}=1 / a+0.8 / b+0.5 / c$. Consequently, we have

$$
\begin{gathered}
F_{R_{\mu}}(\alpha)=\left\{\begin{array}{lll}
\{x, y, z\}, & \text { if } 0 \leqslant \alpha \leqslant 0.5, \\
\{z\}, & \text { if } 0.5<\alpha \leqslant 1 .
\end{array}\right. \\
F_{R_{v}}(\alpha)=\left\{\begin{array}{lll}
\{a, b, c\}, & \text { if } 0 \leqslant \alpha \leqslant 0.5, \\
\{a, b\}, & \text { if } 0.5<\alpha \leqslant 0.8, \\
\{a\}, & \text { if } 0.8<\alpha \leqslant 1 .
\end{array}\right.
\end{gathered}
$$

$F_{R_{\mu}}(\alpha) \times F_{R_{\nu}}(\alpha)=\left\{\begin{array}{lll}U \times V, & \text { if } 0 \leqslant \alpha \leqslant 0.5, \\ \{(z, a),(z, b)\}, & \text { if } 0.5<\alpha \leqslant 0.8, \\ \{(z, a)\}, & \text { if } 0.8<\alpha \leqslant 1 .\end{array}\right.$

$$
F_{R}(\alpha)=\left\{\begin{array}{lll}
U \times V, & \text { if } \quad \alpha=0, \\
U \times V-T_{1}, & \text { if } 0<\alpha \leqslant 0.2, \\
U \times V-T_{2}, & \text { if } 0.2<\alpha \leqslant 0.3 \\
T_{3}, & \text { if } 0.3<\alpha \leqslant 0.5 \\
\{(z, a),(z, b)\}, & \text { if } 0.5<\alpha \leqslant 0.8 \\
\{(z, a)\}, & \text { if } 0.8<\alpha \leqslant 1 .
\end{array}\right.
$$

where $T_{1}=\{(x, a),(y, a)\}, \quad T_{2}=\{(x, a),(y, a),(z, c)\}$ and $T_{3}=\{(x, c),(y, b),(y, c),(z, a),(z, b)\}$.

It follows that $F_{R}(\alpha) \subseteq F_{R_{\mu}}(\alpha) \times F_{R_{v}}(\alpha)$ and $R$ is a soft relation from $R_{\mu}$ to $R_{\nu}$.

\section{Application of soft relations to information systems}

In this section, we consider a typical example of information system given by Pawlak ${ }^{34}$ to illustrate the application of soft relations to data analysis.
Example $6{ }^{34}$ Suppose that there are 900 people which constitute the universe $U$. The population is characterized by the following attributes: Height,Hair, Eyes and Nationality. The information system characterizing this population is given by Table 1 .

Table 1. Characterization of Population

\begin{tabular}{cccccc}
\hline & Height & Hair & Eyes & Nationality & Support \\
\hline$p_{1}$ & tall & blond & blue & Swede & 270 \\
$p_{2}$ & medium & dark & hazel & German & 90 \\
$p_{3}$ & medium & blond & blue & Swede & 90 \\
$p_{4}$ & tall & blond & blue & German & 360 \\
$p_{5}$ & short & red & blue & German & 45 \\
$p_{6}$ & medium & dark & hazel & Swede & 45 \\
\hline
\end{tabular}

Feng et al. ${ }^{35}$ presented a soft attribute analysis approach by using soft truth degrees of elementary soft implications. In particular, the causal relationship between condition attributes Height, Eyes and the decision attribute Nationality is analyzed. Now, we make an analogous analysis on the relationship between condition attribute Hair and the decision attribute Nationality by using soft relations. We hope to know which type of Hair is most important for determining a person's nationality.

According to ${ }^{35}$, we construct the soft sets $(F, A)$ and $(G, B)$ related to attributes Hair and Nationality

\begin{tabular}{|c|c|c|c|c|}
\hline & blond & dark & red & Support \\
\hline$p_{1}$ & 1 & 0 & 0 & 270 \\
\hline$p_{2}$ & 0 & 1 & 0 & 90 \\
\hline$p_{3}$ & 1 & 0 & 0 & 90 \\
\hline$p_{4}$ & 1 & 0 & 0 & 360 \\
\hline$p_{5}$ & 0 & 0 & 1 & 45 \\
\hline$p_{6}$ & 0 & 1 & 0 & 45 \\
\hline \multicolumn{5}{|c|}{ Table 3. Soft set related to "Nationalities } \\
\hline & Swede & \multicolumn{2}{|c|}{ German } & Support \\
\hline$p_{1}$ & 1 & \multicolumn{2}{|c|}{0} & 270 \\
\hline$p_{2}$ & 0 & \multicolumn{2}{|c|}{1} & 90 \\
\hline$p_{3}$ & 1 & \multicolumn{2}{|c|}{0} & 90 \\
\hline$p_{4}$ & 0 & \multicolumn{2}{|c|}{1} & 360 \\
\hline$p_{5}$ & 0 & \multicolumn{2}{|c|}{1} & 45 \\
\hline$p_{6}$ & 1 & \multicolumn{2}{|c|}{0} & 45 \\
\hline
\end{tabular}
respectively in Table 2 and Table 3.

Table 2. Soft set related to "Hair"

By the tabular representations, we have $A=$ $\{\mathrm{bd}, \mathrm{dk}, \mathrm{rd}\}, b=\{\mathrm{sw}, \mathrm{ge}\}$, where $b d, d k, r d$ are stand 
for attribute values blond,dark, red of Hair, sw, ge are stand for attribute values $S$ wede,German of Nationality respectively, and

$$
\begin{aligned}
& F(b d)=\left\{p_{1}, p_{3}, p_{4}\right\}, F(d k)=\left\{p_{2}, p_{6}\right\}, \\
& F(r d)=\left\{p_{5}\right\} . \\
& G(s w)=\left\{p_{1}, p_{3}, p_{6}\right\}, G(g e)=\left\{p_{2}, p_{4}, p_{5}\right\} .
\end{aligned}
$$

We construct a soft relation $(H, A \times B)$ from $(F, A)$ to $(G, B)$ as: for each $a \in A$ and $b \in B$,

$H(a, b)=\left\{\left(p_{i}, p_{i}\right) ; p_{i} \in F(a) \wedge p_{i} \in G(b)\right\}$.

By $H(a, b) \subseteq F(a) \times G(b),(H, A \times B)$ is indeed a soft relation from $(F, A)$ to $(G, B)$. Clearly,

$$
\begin{aligned}
& H(b d, g e)=\left\{\left(p_{4}, p_{4}\right)\right\}, \\
& H(d k, g e)=\left\{\left(p_{2}, p_{2}\right)\right\}, \\
& H(r d, g e)=\left\{\left(p_{5}, p_{5}\right)\right\}, \\
& H(b d, s w)=\left\{\left(p_{1}, p_{1}\right),\left(p_{3}, p_{3}\right)\right\}, \\
& H(d k, s w)=\left\{\left(p_{6}, p_{6}\right)\right\}, \\
& H(r d, s w)=\emptyset .
\end{aligned}
$$

By this soft relation, we can get some information about this population. For example,

(1) The support $|H(b d, g e)|=360,|F(b d)|=720$. $\frac{|H(b d, g e)|}{|F(b d)|}=0.5$ means that $50 \%$ people with blond hair are German.

(2) $\frac{|H(b d, g e)|}{|G(g e)|}=\frac{360}{495}=0.73, \frac{|H(b d, s e)|}{|G(s w)|}=\frac{360}{405}=0.89$. Thus, German are most probably of blond hair, so does Swede.

(3) $\frac{|H(d k, g e)|}{|F(d k)|}=\frac{90}{135}=0.67, \frac{|H(d k, s e)|}{|F(d k)|}=\frac{45}{135}=0.33$. Thus, one can assert that "People of dark hair are probably to be German rather than Swede".

\section{Concluding remarks}

Soft set theory was originally proposed as a general mathematical tool for dealing with uncertainties. Babitha and Sunil ${ }^{30}$ initiated the study of soft set relations and soft set functions. This paper is devoted to a further discussion along this line. The notion of soft relation is proposed which is a generalization of the notion of soft set relation, and some related properties are surveyed. Furthermore, the connections between soft relations and the operations on fuzzy sets are analyzed. It is shown that fuzzy relations and fuzzy soft sets are special cases of soft relations.

In further research, we will consider fuzzy soft relations between fuzzy soft sets in a more general framework. It is expected that the fuzzy soft relations can be established based on fuzzy relations and operations on fuzzy sets. The $t$-norms and $t$-conorms based operations, proposed by Bustince and Burillo ${ }^{36,37}$, on fuzzy sets will play a central role in this study. Moreover, the topology generated by soft relation is also an important and interesting issue to be addressed.

\section{Acknowledgements}

The authors are highly grateful to the anonymous referees for their insightful comments and valuable suggestions which greatly improve the quality of this paper. This work was partially supported by the National Natural Science Foundation of China (Grant No. 61473239, 61175055, 61372187), the Fundamental Research Funds for the Central Universities of China (Grant No. 2682014ZT28), and the open research fund of key laboratory of intelligent network information processing, Xihua University (szjj2014-052).

\section{References}

1. L. A. Zadeh, Fuzzy sets, Information and Control 8(1965)338-353.

2. Z. Pawlak, Rough sets, Int. J. Comput. Inf. Sci. 11(1982)341-356.

3. Z. Pawlak, A. Skowron, Rudiments of rough sets, Inform. Sci. 177(2007)3-27.

4. D. Molodtsov, Soft set theory-First results, Comput. Math. Appl. 37(1999)19-31.

5. D. Molodtsov, The theory of soft sets, URSS Publishers, Moscow(in Russian), 2004.

6. P. K. Maji, R. Biswas and A. R. Roy, Soft set theory, Comput. Math. Appl. 45(2003)555-562.

7. M. Irfan Ali, F. Feng, X. Liu, W. K. Min and M. Shabir, On some new operations in soft set theory, Comput. Math. Appl. 57(2009)1547-1553.

8. A. Sezgin, A. O. Atagun, On operations of soft sets. Comput. Math. Appl. 61(2011)1457-1467.

9. K. Y. Qin and Z. Y. Hong, On soft equality, J. Comput. Appl. Math. 234(2010)1347-1355.

10. Z.Xiao, K.Gong, S.Xia, Y.Zou, Exclusive disjunctive soft sets, Comput. Math. Appl. 59(2010)2128-2137.

11. K.Gong, Z.Xiao, X.Zhang, The bijective soft set with its operations, Comput. Math. Appl. 60(2010)22702278. 
12. H.Aktas, N.Cagman, Soft sets and soft groups, Inform. Sci. 177(2007)2726-2735.

13. F.Feng, Y.B.Jun, X.Z.Zhao, Soft semirings, Comput. Math. Appl. 56(2008)2621-2628.

14. U.Acar, F.Koyuncu, B.Tanay, Soft sets and soft rings, Comput. Math. Appl. 59(2010)3458-3463.

15. Y.B.Jun, Soft BCK/BCI-algebras, Comput. Math. Appl. 56(2008)1408-1413.

16. Y.B.Jun, C.H.Park, Applications of soft sets in ideal theory of BCK/BCI-algebras, Inform. Sci. 178(2008)2466-2475.

17. J.Zhan, Y.B.Jun, Soft BL-algebras based on fuzzy sets, Comput. Math. Appl. 59(2010)2037-2046.

18. P.K.Maji, R.Biswas, A.R.Roy, Fuzzy soft sets, J. Fuzzy Math. 9(3)(2001)589-602.

19. P.Majumdar, S.K.Samanta, Generalised fuzzy soft sets, Comput. Math. Appl. 59(2010)1425-1432.

20. P.K.Maji, R.Biswas, A.R.Roy, Intuitionistic fuzzy soft sets, J. Fuzzy Math. 9(3)(2001)677-692.

21. P.K.Maji, A.R.Roy, R.Biswas, On intuitionistic fuzzy soft sets, J. Fuzzy Math. 12(3)(2004)669-683.

22. X.B.Yang, T.Y.Lin, J.Y.Yang, Y.Li, D.J.Yu, Combination of interval-valued fuzzy set and soft set, Comput. Math. Appl. 58(2009)521-527.

23. W.Xu, J.Ma, S.Wang, G.Hao, Vague soft sets and their properties, Comput. Math. Appl. 59(2010)787-794.

24. Y.Jiang, Y.Tang, Q.Chen, H.Liu, J.Tang, Intervalvalued intuitionistic fuzzy soft sets and their properties, Comput. Math. Appl. 60(2010)906-918.

25. F.Feng, C.X.Li, B.Davvaz, M.I. Ali, Soft sets combined with fuzzy sets and rough sets: a tentative approach, Soft Computing 14(2010)899-911.

26. F.Feng, X.Y,Liu, V.Leoreanu-Fotea, Y.B.Jun, Soft sets and soft rough sets, Inform. Sci. 181(2011)1125-1137.
27. D.Meng, X.H.Zhang, K.Y.Qin, Soft rough fuzzy sets and soft fuzzy rough sets, Comput. Math. Appl. 62(2011)4635-4645.

28. M.I.Ali, A note on soft sets, rough soft sets and fuzzy soft sets, Applied Soft Computing 11(2011)33293332.

29. X.H.Zhang, B.Zhou, P.Li, A general frame for intuitionistic fuzzy rough sets, Inform. Sci. 216(2012)3449.

30. K.V.Babitha, J.J.Sunil, Soft set relations and functions, Comput. Math. Appl. 60(2010)1840-1849.

31. K.V.Babitha, J.J.Sunil, Transitive closures and orderings on soft sets, Comput. Math. Appl. 62(2011)22352239.

32. H.L.Yang, Z.L.Guo, Kernels and closures of soft set relations, and soft set relation mappings, Comput. Math. Appl. 61(2011)651-662.

33. D.Pei, D.Miao, From soft sets to information systems, Proceedings of 2005 IEEE International Conference On Granular Computing, 2005, 617-621.

34. Z.Pawlak, Rough sets, decision algorithms and Bayes' theorem, European J. Operational Research. 136(2002)181-189.

35. F.Feng, M.Akram, B.Davvaz, V.Leoreanu-Fotea, Attribute analysis of information systems based on elementary soft implications, Knowledge-Based Systems 70(2014)281-292.

36. H.Bustince, Construction of intuitionistic fuzzy relations with predetermined properties, Fuzzy Sets and Systems 109(3)(2000)379-403.

37. H.Bustince, P.Burillo, Mathematical analysis of interval-valued fuzzy relations: application to approximate reasoning, Fuzzy Sets and Systems $113(2)(2000) 205-219$. 